\title{
Linx
}

LinX Revue des linguistes de l'université Paris X Nanterre

$76 \mid 2018$

Dire l'humain : les noms généraux dénotant les

humains

\section{Leute (allemand) et gens (français)}

Du nom au pronom?

German Leute vs French gens - from noun to pronoun?

\section{Sarah Rössler}

\section{(2) OpenEdition}

\section{Journals}

Édition électronique

URL : http://journals.openedition.org/linx/2411

DOI : 10.4000/linx.2411

ISSN : 2118-9692

Éditeur

Presses universitaires de Paris Nanterre

Édition imprimée

Date de publication : 31 juillet 2018

Pagination : 115-132

ISSN : 0246-8743

Référence électronique

Sarah Rössler, "Leute (allemand) et gens (français) », Linx [En ligne], 76 | 2018, mis en ligne le 31

janvier 2019, consulté le 20 avril 2019. URL : http://journals.openedition.org/linx/2411 ; DOI : 10.4000/ $\operatorname{lin} x .2411$

Ce document a été généré automatiquement le 20 avril 2019

Département de Sciences du langage, Université Paris Ouest 


\title{
Leute (allemand) et gens (français)
}

\author{
Du nom au pronom? \\ German Leute vs French gens - from noun to pronoun?
}

\section{Sarah Rössler}

\section{Introduction}

1 Si l'on cherche l'équivalent français du nom allemand Leute dans le dictionnaire, on trouve la traduction gens. Il est possible pour les locuteurs natifs allemands d'utiliser le mot français comme ils y sont habitués dans leur langue maternelle. Cela n'est possible que parce que le terme est un plurale tantum dans les deux langues. Ils n'ont pas d'équivalent au singulier, ni en français ni en allemand $\left({ }^{*}\right.$ Leut ou bien * ${ }^{*}$ en $\left.(t)\right)$, même si, autrefois, ces formes ont existé dans les deux langues et que la forme française vient étymologiquement d'un collectif latin au singulier (GDW, TLFi).

Comme Leute et gens sont des noms généraux désignant des humains qui manifestent dans de très nombreuses langues une aptitude à la pronominalisation, il se pose la question de savoir si Leute et gens montrent déjà des signes pour un développement vers un pronom.

Il faut donc examiner si et dans quelle mesure le nom allemand Leute et le nom français gens ont déjà subi un développement vers le pronom : peut-on déjà les définir en tant que pronoms et sous quels aspects montrent-ils des différences en ce qui concerne leur degré de grammaticalisation?

Pour pouvoir saisir l'évolution des termes gens et Leute, il faut examiner plusieurs aspects. On commencera, pour les étudier, par un traitement lexicographique qui donnera les informations les plus élémentaires. Ensuite, on étudiera l'étymologie, qui nous apportera des informations sur les radicaux de gens et Leute. En analysant des aspects comme la possibilité d'être comptable, le genre, les possibilités de composition et les restrictions référentielles, il sera possible de connaître les différences et points communs existant entre les deux mots. La comparaison des résultats montrera si et dans quelle mesure les deux noms ont déjà progressé dans leur processus d'évolution vers la valeur pronominale. 


\section{Leute et gens : traitement lexicographique}

5 Gens et Leute se ressemblent à certains égards : les deux mots étaient utilisés au MoyenÂge en tant que collectif et référaient au peuple. La langue allemande, tout comme la langue française, réfère à un groupe d'hommes par un plurale tantum. Les définitions des dictionnaires convergent pour dire que Leute désigne " eine mit anderen zusammen auftretende, als Menge o. Ä. gesehene Menschen » (Duden Online, s.v. Leute), donc des gens qui apparaissent ensemble en tant qu'une foule. Leute réunit les participants d'un groupe et on trouve au contraire la désignation " Menschen ", qui souligne l'existence des individus. Il y a aussi une utilisation vieillie qui réfère aux employés de maison, aux valets et aux ouvriers. A partir de cette connotation se sont développés aussi de nombreux mots composés.

6 Leute peut aussi décrire des personnes en relation avec leur provenance : Stadtleute (" gens de la ville ») ou Dorfleute (" gens du village ») désignent les personnes en général. Landsleute ("gens du pays ») est tout à fait courant et réfère aux gens qui viennent du même pays. Le dictionnaire inverse de Lee (2005) mentionne 63 mots composés en -leute issus de domaines différents. On trouve par exemple Bergleute («mineurs »), Brautleute («mariés»), Eheleute («conjoints»), Kaufleute («commerçants») et Nachbarsleute (« voisins »). La tête Leut- n'existe pas, comme la pure forme singulière n'existe plus.

7 Grimm et Grimm (1885) mentionnent que Leute peut s'utiliser pour s'adresser à quelqu'un. Cette fonction est encore usitée aujourd'hui : même après 130 ans, Hey Leute est toujours en usage dans la langue des jeunes.

8 Le mot français gens est défini dans le TLFi comme " personnes en nombre indéterminé, considérées collectivement ». Les synonymes sont, curieusement, selon Robert (Robert et al., 1984) des formes singulières comme homme, humain et personne. Malgré son sens collectif et sa forme au pluriel, le mot gens peut référer à une personne connue ou au locuteur lui-même. C'est le cas dans les énoncés (1) et (2) :

(1) On ne laisse pas tomber les gens comme ça.

(2) Vous avez une manière de parler aux gens! (Dubois, 1966, s.v. gens)

En français, on trouve également l'utilisation de gens pour dénommer des noms de fonctions. Ces noms existent à côté des termes techniques.

(3) les gens d'Église ( les ecclésiastiques, les prêtres »)

(4) les gens de lettres (« les écrivains, les auteurs»)

Comme en allemand, on peut désigner des personnes qui ont une certaine relation par gens en combinaison avec un déterminant possessif. Par ce moyen, on exprime des relations unissant des partisans ou des parents, ce qui montre encore une fois des parallèles avec l'allemand.

11 Pendant la Guerre de cent ans s'est développée l'expression de gens d'armes qui désignait des cavaliers armés et qu'on peut trouver encore aujourd'hui dans gendarme (au singulier !) et gendarmerie.

\section{L'étymologie de Leute et gens}

12 Le nom allemand Leute est dérivé du nom du moyen-haut-allemand liute et lui-même provient de l'ancien haut-allemand liuti. En ancien haut-allemand, il existait une forme au 
singulier dër liut qui désignait le peuple en général. Avec l'adoption de la forme grammaticale dans son sens pluriel se formait le terme liuti en moyen-haut-allemand (cf. Paul, 1917 : 102). Le collectif au singulier existe encore dans quelques dialectes allemands. On trouve par exemple en bavarois l'expression « das mannete/weibete Leut » («la gent masculine » et féminine ») (cf. Grimm et Grimm 1885). La grammaire allemande de Paul (1917) ainsi que le dictionnaire français de Dauzat \& alii (1973) font mention de l'évolution parallèle des pluralia tantum français et allemands.

La base du nom français actuel gens est le latin gens, gentis qui désignait une tribu, des citoyens ou des membres d'une famille. Le sens collectif lors d'une utilisation au singulier s'ébauche déjà en latin. Le pluriel gentes avec la signification de "gens, hommes " se manifeste en latin classique du premier siècle, par exemple chez le poète Horace (cf. Wartburg, 1967, s.v. gens).

A partir du pluriel collectif, il s'est formé un nouveau singulier qui porte la signification de «individu, personne» et qui accepte d'abord la combinaison avec des adjectifs numéraux. La langue écrite cependant n'a pas maintenu cette fonction et le caractère nombrable est devenu agrammatical à la fin du $17^{\grave{e ̀ ~}}$ siècle (ibid). Au début du $19^{\mathrm{è}}$ siècle, gens s'est figé dans la forme de plurale tantum et cette utilisation est devenue de plus en plus courante jusqu'au 20 è siècle (Cappeau et Schnedecker, 2012:5).

On peut donc constater que le développement des deux termes s'est déroulé de façon parallèle et qu'il y a des aspects communs, malgré les racines différentes, entre gens et Leute. Dans les deux langues, il y a eu temporairement une forme au singulier; l'ancien haut-allemand utilisait liut déjà en tant que pur collectif, tandis que l'ancien français disposait d'une signification de singulier et de pluriel. Le terme allemand aussi bien que que le terme français comprennent le sens "peuple", mais dans les deux langues on trouve aussi simultanément un synonyme correspondant.

\section{Gens et Leute entre singulare tantum et plurale tantum, et entre nom comptable et nom collectif}

Le terme allemand Leute ainsi que le français gens réfèrent à une multiplicité d'entités qui conceptualisent un ensemble.

Le terme allemand Leute ainsi que le français gens réfèrent à une multiplicité d'entités qui conceptualisent un ensemble. Nous caractérisons intuitivement un groupe d'hommes comme gens. L'analogie du français et de l'allemand est frappante. Le même phénomène se manifeste aussi en anglais qui utilise également un collectif people. Le français diffère des autres langues romanes qui utilisent un nom collectif au singulier : en espagnol la gente, en italien la gente et en portugais a gente (aujourd'hui pronominalisé). Il faut donc examiner les caractéristiques de la pluralité des deux noms. La classification comme plurale tantum a pour conséquence que la terminaison du pluriel -e est déjà fossilisée et qu'elle appartient ainsi à la racine du mot Leute (cf. Lang, $2000: 19)$. Cela est aussi valable pour le pluriel $-s$ du mot français gens.

18 Leute et gens réfèrent à un groupe d'hommes formant une unité compacte. C'est ce qui distingue Leute de Menschen.

(5) Drei Menschen stehen vor der Tür.

(6) Drei Leute stehen vor der Tür. 
19 On peut comprendre l'exemple (5) comme suit : trois hommes sont devant la porte, mais indépendants l'un de l'autre. L'exemple (6) fait ressentir un regroupement des trois hommes et montre ainsi le statut de collectif de groupe de Leute (cf. Mihatsch, $2000: 40$ ). Il est frappant que la combinaison des différents noms n'est pas acceptable et qu'une énumération n'est pas possible:

(7) Auf dem Bild sind *eine Person und zwei Leute, also drei Menschen.

(« Sur l'image, il y a *une personne et deux gens, donc trois hommes »)

(8) Auf dem Bild sind drei Personen: *ein Mensch und zwei Leute (exemples de

Lang, 19) («Sur l'image, il y a trois personnes : *un homme et deux gens »)

Le problème de la combinaison des $\mathrm{N}$ est qu'ils ont des spécifications différentes. On peut constater que Person désigne un individu, que Mensch établit un contraste avec un animal et que Leute comprend un groupe de personnes et se démarque des autres noms par son statut de plurale tantum. Pour cette raison, le lien entre les trois termes suscite un jugement d'inacceptabilité de cette association. Leute se réfère intrinsèquement au genre humain, à tel point qu'une démarcation contrastive n'est pas possible de cette manière. On constate qu'en français, cette combinaison n'est pas davantage possible.

Dans la pratique, on peut compter les composants qui forment Leute. Les pluralia tantum se démarquent ainsi des noms de masse (comme eau ou farine) dont les composants ne peuvent pas être reconnus comme individus (cf. Wisniewski, $2010: 184$ ). On constate que :

(9) gens + gens $=($ plus de) gens

22 A cet égard, le $\mathrm{N}$ ressemble aux noms de masse : les composants ne sont plus importants (pour l'addition des noms de masse, voir aussi Kleiber, 1987 : 119). A partir d'un nombre de deux, des hommes peuvent être désignés comme Leute. L'exemple (6) nous montre qu'une individualisation des composants est possible sans problème. Il s'agit donc d'un pluriel discontinu, dans lequel les composants sont comptables. Le pluriel discontinu ne permet pas de démarcation lors d'un accroissement du matériel, comme c'est aussi le cas pour les noms de masse (Gréa, 2012 : 194).

On peut déterminer le nombre exact de Leute, mais cela n'est pas important, car c'est un nom collectif. Si le locuteur voulait isoler les individus, il choisirait un autre terme. Gréa (ibid.) exprime le phénomène comme suit : "La discontinuité entre les atomes de la pluralité s'efface au profit du tout et de son unité ». Il s'agit donc de l'appartenance à un groupe dont le locuteur ne connaît ni les membres ni le nombre et qui, par conséquent, ne sont comptables qu'en théorie.

(10) Tous les gens qui aiment regarder le foot.

Dans ce cas, le locuteur ne peut pas spécifier à qui il se réfère. En outre, les membres du groupe ne doivent pas forcément se connaître. L'appartenance est créée par le locuteur, par exemple par le même intérêt de tous les membres du groupe de s'intéresser au foot. L'appartenance au groupe est limitée dans son contexte, dans l'espace et le temps (cf. Mihatsch, 2015b : 88). Pour cette raison on peut considérer que Leute/gens possédent des propriétés de stage level et non pas de individual level.

Il se pose donc la difficulté de subdiviser le groupe des gens en individus :

(11) ??Les gens sont arrivés l'un après l'autre (exemples de Cappeau et Schnedecker, 2014 : 3038).

Alors qu'en français l'acceptabilité de cette tournure est douteuse, elle est tout à fait courante en allemand :

(12) Die Leute sind einer nach dem anderen eingetroffen. 
montre déjà que Leute accepte la combinaison avec des numéraux, ce qui permet une individualisation des membres du groupe. Cette différence souligne que le français conceptualise le terme d'une autre manière que l'allemand. En français, le groupe est inséparable.

La plus grande différence entre gens et Leute réside dans la quantification des termes. En allemand il n'est pas problématique de référer à drei Leute (voir exemple (6)). Cette combinaison n'est pas possible en français contemporain. Mais il n'en a pas toujours été ainsi. Jusqu'au $18^{\mathrm{è}}$ siècle, gens pouvait être nombré :

(13) dix, vingt, ... gens (Robert et al., 1984)

Aujourd'hui, on ne peut plus lier gens à un numéral; cependant, l'utilisation en combinaison avec une valeur approchée, est possible :

(14) une douzaine, centaine, ... de gens (ibid)

L'utilisation avec un numéral est possible quand gens est spécifié par un adjectif :

(15) trois pauvres gens / quatre honnêtes gens

L'allemand ne connaît pas cette restriction. La combinaison est tout à fait courante et ne pose pas de problèmes dans la réalité. Comme les hommes peuvent pratiquement être comptés, on trouve souvent, surtout dans le langage courant, des expressions comme :

(16) Es waren 25.000 Leute im Stadion (il y avait 25.000 gens dans le stade.)

En français ainsi qu'en allemand la combinaison avec des quantificateurs indéfinis est possible : beaucoup de gens, la plupart des gens ou viele Leute, die meisten Leute (pour plus d'informations voir Mihatsch, 2015b : 88). La différence dans la possibilité de compter les termes montre une différence de conceptualisation en français et en allemand. En français, gens est conceptualisé comme collectif à un plus haut degré que l'allemand Leute. En allemand, une individualisation est possible et les membres du groupe peuvent être comptés séparément. Par là, on peut comprendre Leute comme une pluralité d'individus, même si la formation d'un singulier n'est pas possible. Le français se montre plus figé et ne permet pas, même dans le langage courant, de compter des gens. La langue française doit recourir à un niveau de langue supérieur, par exemple personnes, pour pouvoir référer un nombre exact des individus d'un groupe.

Cet aspect du français constitue la plus grande différence entre Leute et gens, et souligne la compréhension de gens comme un groupe compact, tandis que l'allemand autorise encore la possibilité de compter les individus du groupe.

\section{Les changements de genre}

L'ancien français possède, ainsi que l'ancien haut-allemand, une forme au singulier, la gent, qu'on trouve encore aujourd'hui, par analogie avec le latin, au féminin. SaintePalaye (1972) constate qu'au Moyen-Âge encore, la forme au pluriel était aussi de genre féminin. Le sens sémantique de la gent était collectif avec la signification de "peuple, nation, troupe » ou désignait un individu " personne, homme » (Godefroy, 2006, s.v. gent). La gent n'existe que pour un certain temps en tant que non-collectif (Grevisse et Goosse, 2006, s.v. gent.). Bloch et Wartburg constatent que gent n'avait plus qu'une utilisation littéraire au $17^{\mathrm{è}}$ siècle. Quelques écrivains, tel La Fontaine, ont essayé de maintenir la forme, mais ne réussirent pas à la faire adopter. La gent peut encore être trouvée dans 
quelques dialectes d'aujourd'hui, par exemple en provençal una gent avec la signification de une personne (Honnorat, 1971, s.v. gent).

A cause de la signification collective de gent, on observe souvent un accord par syllepse (ou ad sensum) avec un verbe au pluriel. (cf. Wartburg, 1967). La signification de gens a subi un développement au sens de « hommes » en général, et dès lors le mot a désigné les individus aussi bien masculins que féminins. A partir $\mathrm{du} 13^{\mathrm{e}}$ siècle, on trouve le masculin qui remplace progressivement le genre féminin de gent/gens.

Il est remarquable que gens a maintenu l'indétermination en ce qui concerne le genre. Dubois (1966) donne la définition « $\mathrm{n}$. $\mathrm{m}$. et $\mathrm{f}$. pl. ». Les adjectifs placés devant gens, sont utilisés au féminin et déterminent ainsi le genre du nom. Tous les adjectifs et autres termes postposés à gens restent au masculin, comme par exemple :

(17) de vieilles gens

(18) les gens gais (ibid)

La combinaison tous les gens fait exception, où tous reste au masculin même s'il est antéposé. On remarque que le français d'aujourd'hui maintient les deux genres. En allemand, il n'y a pas à faire un accord de l'adjectif qui pourrait indiquer le genre, et Duden Online (2015) ne donne pas de genre pour le « Pluralwort » Leute.

\section{Leute et gens comme éléments de composition}

L'allemand et le français comportent un grand nombre de syntagmes ou de mots composés plus ou moins lexicalisés à base " gens » et « Leute ».

Les dictionnaires consultés contiennent très peu d'entrées de ce type. Ce sont les noms de métiers comme gens de mer, gens de lettres ou gens d'Eglise (cf. Dubois et Dubois-Charlier, 1996). Il est frappant que parmi les dictionnaires français consultés, seul le TLFi (TLFi, s.v. gens) fait une liste détaillée de mots composés. Il y a une catégorie " gens de + subst. ». On trouve différentes désignations : selon le statut ("gens du roi »), la qualité ("gens de bien »), l'activité (« gens de service »), la provenance (« gens de Galice »), la classe sociale (" gens de rien », " gens de cour »).

Pour trouver les mots composés allemands, il faut consulter un dictionnaire inverse. Le dictionnaire consulté (Lee, 2005) donne une quantité de lemmes. Etant donné le grand nombre de mots composés allemands avec -leute et le petit nombre de mots composés français, on peut conclure que le français n'a pas lexicalisé des mots composés avec gens de $x$. Comme les mots français ne se composent pas de la même façon que les mots allemands, la reconnaissance des mots composés n'est pas aussi facile en français qu'en allemand.

Lang (2000) constate que les mots composés ne suivent pas les restrictions de généricité, de prédicativité ou de topicalité. Les exemples (19) et (20) montrent cette différence :

(19) *Anna und Peter sind Leute.

(20) Anna und Peter sind Bergleute.

La spécification par la composition fait que le $\mathrm{N}$ peut être l'attribut dans la phrase. Cela n'est pas possible pour le mot simple Leute n'apportant pas de spécifications. Tous les hommes sont des gens, il n'est donc pas pertinent de les désigner en tant que tels. A ce point se manifeste la non-généricité, par comparaison avec Menschen :

(21) Anna und Peter sind Menschen. 

d'ajouter une proposition relative, un adjectif ou d'autres compléments.

(22) *Louise et Pierre sont des gens.

(23) Louise et Pierre sont des gens de mer. qu'un suffixoïde : Jäger porte la signification et -leute n'ajoute que la marque du pluriel. Le mot composé Kaufleute n'a pas cette fonction de suffixoïde, puisque le seul Kauf- ne porte pas de signification et a besoin de -leute pour ajouter la spécification /+hum/ (cf. Lang, 2000 : 42). A cause de sa valeur sémantique insignifiante, -leute peut être considéré comme terminaison de pluriel lexical : -leute prend le statut d'un suffixe collectif qui marque la pluralité d'une manière neutre.

49 Ce phénomène de la supplétion est spécifique à l'allemand et ne peut pas être comparé au français. A cause de la structure de la formation de mots, le français ne peut pas composer des mots avec un changement de la signification.

\section{Les positions syntaxiques de Leute et gens}

Leute ainsi que gens peuvent être utilisés avec l'article défini et indéfini. Comme mentionné supra, une spécification est nécessaire :

(24) J'ai rencontré des gens qui ont chanté.

(25) Ich habe ø Leute getroffen, die gesungen haben.

(26) J'ai rencontré les gens qui ont chanté. 
(27) Ich habe die Leute getroffen, die gesungen haben. homme, individu ou humain. Dans les dictionnaires allemands on trouve des synonymes Menschen (« hommes »), mais aussi Bevölkerung (" population »), Bürger (« citoyens ») et die (breite) Masse («la foule»). La référence à Angestellte (" employés»), Personal («personnel») et Familienangehörige (cf. Scholze-Stubenrecht, 2011, s.v. Leute ) (« membres de la famille ») est aussi donnée. Les analogues synonymes sont similaires en français. Les caractéristiques des synonymes (pour plus d'informations sur les synonymes de Leute, voir Mihatsch 2015b) empêchent la substituabilité entre eux.

« Leute sind immer Menschen, aber Menschen sind nicht immer Leute » (Lang, $2000: 12$ ). «Des gens sont toujours des hommes, mais des hommes ne sont pas toujours des gens »: autrement dit: Leute et Menschen ne sont que des quasi-synonymes. Leute et gens ne peuvent pas référer au genre humain, ce qu'on peut aussi expliquer par leur statut de 
stage level predicate. Pour pouvoir désigner le genre humain, on a besoin d'un individual level predicate. Un autre obstacle est la nécessité de la spécification de gens.

(33) *Adam und Eva waren die ersten Leute (exemple de Lang, $2000: 13)$.

(34) *Adam et Eve étaient les premiers gens.

Malgré la spécification par "premier », on ne peut pas utiliser le $\mathrm{N}$ en tant qu'attribut. Par l'utilisation de Leute et gens, on ne crée pas de message portant un sens et l'auditeur attend inévitablement une suite sous forme de proposition relative. Si Leute apparait seul, il définit la personne de référence seulement comme /+humain/ et cela n'est pas suffisant pour une désignation générique.

L'exemple (35) montre que Leute/gens au pluriel sans spécification est non-générique :

(35) *Wir sind alle Leute (exemple de Lang, $2000: 15)$.

(36) *Nous sommes tous des gens.

La caractéristique être des gens ne peut pas séparer des individus du grand groupe des hommes (cf. Lang, 2000:15). Pour cette raison, la formulation suivante nous parait bizarre dans les deux langues :

(37) Die Menschen sind komische Leute (cf. Lang, $2000: 15$ ).

(38) ?'Les hommes sont des gens bizarres.

On fait référence au sujet clairement générique les hommes par un attribut non-générique. La contrainte syntaxique des deux $\mathrm{N}$ opposés nous fait sentir la particularité de la phrase.

On remarque que Leute/gens n'est pas une sous-catégorie qui peut être considérée comme une partie de Menschen/hommes. Une classification taxonomique n'est donc pas possible.

En plus, Leute ainsi que gens ne peuvent former que très peu de dérivations. Comme déjà montré, le collectif est restreint à cause de ses caractéristiques sémantiques et morphologiques. La liste des 52 exemples chez Grimm et Grimm (1885) est désuète et évoque des circonstances sociales qui n'existent plus. Des lemmes comme Leutebeschmeiszer («quelqu'un qui jette quelque chose sur des gens»), Leuteschlächter (« un boucher des gens»), Leutfresser (« quelqu'un qui mange des gens») ou Leuttäuscher ("quelqu'un qui trompe les gens») ne sont plus imaginables aujourd'hui. On peut souligner qu'ici le -e du pluriel n'apparaît pas dans quelques formes composées. Il y a quelques centaines d'années, l'allemand avait encore plus de possibilités de former des mots à cause de la signification de "valets " pour Leute. Même si le français connaissait aussi cette connotation, les dictionnaires comprennent beaucoup moins d'exemples.

Il n'est pas possible non plus de former des adjectifs ou des verbes avec Leute, par exemple analogue à Menschen :

(39) menschenleer («sans hommes»), menschlich («humains»), menscheln («montrer de l'humanité »)

(40) *leuteleer, *leutlich, *leuteln

Le statut de plurale tantum a donc des effets limitatifs pour Leute et gens en ce qui concerne la composition et la dérivation. Compte-tenu des racines différentes des deux langues, les points communs sont étonnants.

\section{Conclusion}

Après avoir analysé les deux noms d'humains gens et Leute depuis le début de leur existence jusqu'à aujourd'hui, nous constatons des parallèles étonnants entre le français et l'allemand. La perte de quelques fonctions que le nom possédait encore il y a quelques 
siècles montre que le nom se fige peu à peu. Comme les deux termes ne peuvent plus être utilisés en tant que substantifs habituels, on peut conclure qu'une grammaticalisation partielle a déjà eu lieu. Comparée avec celle d'autres noms d'humains comme tous ou tout le monde, la grammaticalisation de gens et Leute est moins avancée.

Dans les deux langues le terme, après avoir été utilisé au singulier, s'est figé sous forme d'un plurale tantum. Sur le plan sémantique, on a rencontré un changement de la signification, au cours duquel la signification d'employé a été remplacée par la référence à n'importe quel groupe humain. Gens et Leute n'ont qu'un signifié restreint, puisqu'ils ne peuvent désigner qu'un groupe spécifique d'hommes. Dans plusieurs domaines, leurs possibilités d'utilisation sont limitées. La réduction au plurale tantum peut être un pas en direction du pronom, mais cela n'est pas obligatoire. Dans quelques cas, gens peut être remplacé par un pronom. Leute peut donc remplacer tous les termes qui désignent une pluralité humaine, sauf l'humanité.

Voici un aperçu des propriétés des deux noms :

\begin{tabular}{|l|l|l|}
\hline & gens & Leute \\
\hline plurale tantum & oui & oui \\
\hline singulier ancien & oui & oui \\
\hline noms de fonction & oui & oui \\
\hline non-générique & oui & oui \\
\hline comptable & non & oui \\
\hline référence à un groupe spécifique à la 3e personne & oui & oui \\
\hline spécification nécessaire & oui & oui \\
\hline genre indéterminé & oui & oui \\
\hline composition possible & limité & oui \\
\hline formation de dérivations possible & limité & limité \\
\hline échange libre avec synonymes & non & non \\
\hline
\end{tabular}

Tableau 1

72 Ce tableau montre que les deux termes se ressemblent dans la plupart des catégories examinées. Les différences dans la composition ont leur origine dans la façon de la langue française de former des mots composés et ce n'est pas une conséquence des caractéristiques du mot gens. 


\section{BIBLIOGRAPHIE}

BAIDER, F, (im Druck), « Noms génériques 'être humain masculin' et 'être humain féminin' impossibilité sociologique de la modélisation sémantique », dans W. Mihatsch, C. Schnedecker (eds), NHUMA Sammelband, p. 145-176.

BEYER, H., BEYER, A., 1987, Sprichwörterlexikon. Sprichwörter und sprichwörtliche Ausdrücke aus deutschen Sammlungen vom 16. Jahrhundert bis zur Gegenwart, München, Beck.

BLOCH, O., VON WARTBURG, W., 1968, Dictionnaire étymologique de la langue française, Paris, Presses Univ. de France.

BRAUN, P., 1997, Personenbezeichnungen. Der Mensch in der deutschen Sprache, Tübingen, Niemeyer.

BRAUN, W., 1989, Etymologisches Wörterbuch des Deutschen, Berlin, Akad.-Verl.

BRAUNMÜLLER, K., 1977, Referenz und Pronominalisierung. Zu den Deiktika und Proformen des Deutschen, Tübingen, Niemeyer.

BUßMANN, H., GERSTNER-LINK, C. (eds), 2002, Lexikon der Sprachwissenschaft, Stuttgart, Kröner.

CAPPEAU, P., SCHNEDECKER, C., 2012, « (Les/des) gens vs (les/des) personnes évolution diachronique et comparaison oral / écrit. Des SN en voie de pronominalisation? ", dans K. Jeppesen Kragh, J. Lindschouw (eds), Les variations diasystématiques et leurs interdépendances dans les langues romanes. Actes du Colloque DIA II à Copenhague (19-21 nov. 2012).

CAPPEAU, P., SCHNEDECKER, C., 2014, « Gens, personne(s), individu(s): Trois saisies de l'humain ", dans SHS Web of Conferences, 8, p. 3027-3040.

CARLSON, G. N., 1980, Reference to kinds in English, New York, Garland.

CRESTI, E., MONEGLIA, M., 2005, C-ORAL-ROM. Integrated Reference Corpora for Spoken Romance Languages, Amsterdam/ Philadelphia, John Benjamins Publishing Company.

DAUZAT, A., DUBOIS, J., MITTÉRAND, H., 1973, Nouveau dictionnaire étymologique et historique, Paris, Larousse.

DUBOIS, J., 1966, Dictionnaire du français contemporain, Paris, Larousse.

DUBOIS, J., DUBOIS-CHARLIER, F., 1996, « Collectifs d'êtres vivants », dans LINX, 34/ 5, p. 125-132.

Duden Online, 2015, Duden Online,<http://www.duden.de/>

DUNETON, C., CLAVAL, S., VIMARD, N., 1990, Le bouquet des expressions imagées. Encyclopédie thématique des locutions figurées de la langue française, Paris, Éd. du Seuil.

EISENBERG, P., WERMKE, M. (eds), 2007, Duden - richtiges und gutes Deutsch. Wörterbuch der sprachlichen Zweifelsfälle; auf der Grundlage der neuen amtlichen Rechtschreibregeln ; [Antwort auf grammatische und stilistische Fragen, Formulierungshilfen und Erläuterungen zum Sprachgebrauch], Mannheim, Dudenverl.

GODEFROY, F., 2006, Dictionnaire de l'ancienne langue française, <http://www.classiquesgarnier.com/numerique-bases/index.php?

module=App\&action=FrameMain\&colname=ColGodefroy $>$ 
GRÉA, P., 2012, « Deux-trois mots» sur les déterminants de petite quantité: Pluriel continu et perception sémantique », dans Journal of French Language Studies, 23/2, p. 193-219.

GREVISSE, M., CHAMSON, A., 1982, Le Français correct. Guide pratique, Paris, Duculot.

GREVISSE, M., GOOSSE, A., 2006, Le bon usage. Grammaire française, Paris, Duculot.

GRIMM, J., GRIMM, W., 1885, Deutsches Wörterbuch, Bd. 6, L, M, Leipzig, Hirzel.

GROSS, G., 2008, « Sur le statut des substantifs humains », dans D. Leeman (ed), Des topoï à la théorie des stéréotypes en passant par la polyphonie et l'argumentation. Hommages à Jean-Claude Anscombre, Chambery, Université de Savoie, p. 27-41.

HONNORAT, S. J., 1971, Dictionnaire provençal-français ou Dictionnaire de la langue d'oc ancienne et moderne. Suivi d'un vocabulaire français-provençal, Bd. 2, Genève, Slatkine.

KEMPCKE, G., 1984, Handwörterbuch der deutschen Gegenwartssprache, 2 Bd., Berlin, Akad.-Verl.

KLEIBER, G., 1987, « Mais à quoi sert donc le mot chose? [Une situation paradoxale.] », dans Langue française, 73, p. 109-128.

KLUGE, F., MITZKA, W., 1960, Etymologisches Wörterbuch der deutschen Sprache, Berlin, de Gruyter.

LANG, E., 2000, « Menschen vs. Leute: Bericht über eine semantische Expedition in den lexikalischen Nahbereich », dans U. Kramer (ed), Lexikologisch-lexikographische Aspekte der deutschen Gegenwartssprache. Symposiumsvorträge, Berlin 1997, Tübingen, Niemeyer, p. 1-46.

LEE, D., 2005, Rückläufiges Wörterbuch der deutschen Sprache, Berlin, de Gruyter.

LEHMANN, C., 1995, Thoughts on grammaticalization, München i.e. Unterschleissheim, LINCOM Europa.

LEHMANN, C., 2009, Christian Lehmann, <http://www.christianlehmann.eu/>.

MACKENSEN, L. (ed), 1970, Deutsches Wörterbuch. Rechtschreibung, Grammatik, Stil, Worterklärung, Fremdwörterbuch, München, Südwest-Verl.

MALOUX, M., 1960, Dictionnaire de proverbes, sentences et maximes, Paris, Larousse.

MIHATSCH, W., 2000, « Wieso ist ein Kollektivum ein Kollektivum?: Zentrum und Peripherie einer Kategorie am Beispiel des Spanischen », dans Philologie im Netz, 13, p. 39-72.

MIHATSCH, W., 2006, Kognitive Grundlagen lexikalischer Hierarchien. Untersucht am Beispiel des Französischen und Spanischen, Tübingen, Niemeyer.

MIHATSCH, W., 2013, « Collectives », dans P. O. Müller, I. Ohnheiser, S. Olsen, F. Rainer (eds), HSK word-formation an international handbook of the languages of Europe, Berlin, de Gruyter, p. 1139-1151.

MIHATSCH, W., 2015, « La position taxinomique et les réseaux méronymiques des noms généraux 'être humain' français et allemands ", dans C. Schnedecker, W. Mihatsch (eds), Les noms d'humains une catégorie à part?, Stuttgart, Franz Steiner, p. 70-96.

MIHATSCH, W., 2015, « La sémantique des noms généraux 'être humain' français et allemands », dans C. Schnedecker, W. Mihatsch (eds), Les noms d'humains une catégorie à part?, Stuttgart, Franz Steiner Verlag, p. 44-69.

PAUL, H., 1917, Deutsche Grammatik. Bd. II, Teil 3, Flexionslehre, Tübingen, Niemeyer.

REY-DEBOVE, J., REY, A., 2014, Le Petit Robert de la langue française 2014. Grand format, Paris, Le Robert. 
ROBERT, P., REY, A., REY-DEBOVE, J., 1984, Dictionnaire alphabétique et analogique de la langue française. Tome troisième, Paris, Le Robert.

SAINTE-PALAYE, J. B. de, 1972, Dictionnaire historique de l'ancien langage François ou Glossaire de la langue Françoise, Hildesheim, Olms.

SCHMITT, C., 1993, « Ausgangssprachliche Produktivität und zielsprachliche Aktivität in der Wortbildungslehre: Zu den französischen Entsprechungen deutscher Kollektivmorpheme », dans K. J. Mattheier, K. P. Wegera, W. Hoffmann, H. J. Solms (eds), Vielfalt des Deutschen. Festschrift für Werner Besch, Frankfurt am Main, Lang, p. 533-549.

SCHNEDECKER, C., 2012, « Tout le monde, tous, (tous) les gens Relations sémantique entre des expressions dénotant la totalité /+hum/ ", dans N. Le Querler, F. Neveu, E. Roussel (eds), Relations, connexions, dépendances. Hommage au professeur Claude Guimier [textes des communications présentées lors du colloque organisé à Caen, 17-18 mars 2011, Rennes, Presses universitaires de Rennes, p. 127-155.

SCHOLZE-STUBENRECHT, W. (ed), 2011, Duden - Deutsches Universalwörterbuch. [das umfassende Bedeutungswörterbuch der deutschen Gegenwartssprache mit mehr als 500.000 Stichwörtern, Bedeutungsangaben und Beispielen; rund 250.000 zusätzlichen Angaben zu Grammatik, Herkunft und Stil ; einer tabellarischen Kurzgrammatik der deutschen Sprache], Berlin, Dudenverl.

SZCZEPANIAK, R., 2009, Grammatikalisierung im Deutschen. Eine Einführung, Tübingen, Narr.

TLFi = Trésor de la langue française informatisé, 2002, <http://atilf.atilf.fr/tlf.htm>

WANDER, K. F. W. (eds), 1964, Deutsches Sprichwörter-Lexikon. Ein Hausschatz für das deutsche Volk, Bd. 3, Darmstadt, Wiss. Buchgesellschaft.

WARTBURG, W. von, 1967, Französisches etymologisches Wörterbuch. Eine Darstellung des galloromanischen Sprachschatzes, Basel, Zbinden.

WISNIEWSKI, E. J., 2010, « On using count nouns, mass nouns and pluralia tantum: What counts? ", dans F. J. Pelletier (ed), Kinds, Things, and Stuff: Mass Terms and Generics, New York, Oxford University Press, p. 166-190.

WORTSCHATZ LEIPZIG, Wortschatz-Leipzig, <http://wortschatz.uni-leipzig.de/abfrage/>

\section{RÉSUMÉS}

Les noms Leute et gens désignent un groupe plus ou moins déterminé de personnes. La langue française ainsi que la langue allemande utilisent un plurale tantum et représentent donc un cas particulier parmi les langues qui nous entourent. Comparé aux autres langues européennes, le français et l'allemand montrent des parallèles étonnantes concernant ces deux noms. L'analyse montre qu'ils ont souvent les mêmes restrictions. Ce fait soulève la question de la comparabilité des deux termes concernant leur statut actuel d'un nom humain ou d'un pronom développant. J'examinerai quelles différences il y a entre le mot allemand Leute et le mot français gens et dans quelle mesure les noms se sont déjà développés en direction des pronoms.

The nouns Leute and gens designate a more or less determined group of persons. In French as well as in German speakers use a plurale tantum and in this they represent a special case among the languages around us. In comparison with the other European languages, German and French show surprising parallels for these two nouns. The analysis shows that they often have the same restrictions. This fact throws up the question of the comparability of the two terms concerning their current state as a human noun or as a developing pronoun. I will investigate which 
differences there are between the German word Leute and the French word gens and to what extent they have already developped in the direction of pronouns.

INDEX

Mots-clés : noms généraux d'humains, mot « Leute », mot « gens »

\section{AUTEUR}

\section{SARAH RÖSSLER}

Université de Bochum (Allemagne) 